Editorial

\title{
Impact of COVID-19 Pandemic on Cancer Screening Services
}

\author{
Gambhir Shrestha ${ }^{1}$, Rashmi Mulmi \\ ${ }^{1}$ Department of Community Medicine, Maharajgunj Medical Campus, Institute of Medicine, Tribhuvan University, \\ Kathmandu, Nepal \\ ${ }^{2}$ Department of Cancer Prevention, Control and Research, B.P. Koirala Memorial Cancer Hospital, Bharatpur, Nepal
}

Coronavirus disease 2019 (COVID-19) first reported from China on 31 December 2019 and declared "public health emergency of international concern" by World Health Organization on 30 January 2020 and pandemic on 11 March $2020 .^{1}$ The Government of Nepal imposed a nationwide lockdown since 24 March 2020 till 15 June 2020 , and thereafter there has been some relaxation and again strict lockdown in some places. ${ }^{2}$ Public health measures such as social distancing, travel restrictions, and quarantine/isolation have been introduced. The patients were advised only to visit the hospital if urgency and the health providers postponed non-urgent cases. COVID-19 pandemic has disrupted regular health care services including cancer screening. Additionally, changes in health-seeking behavior, constrained physical access, and financial hardship have also limited service uptake. This new pandemic has challenged the health system in delivering both the emergency and routine health services.

Routine screening of patients without symptoms plays an important role in the early diagnosis of various cancers, including breast, colorectal, oral, and cervical cancers. However, the COVID-19 pandemic has caused many institutions to pause their screening activities. In the current situation, cancer screening may not be of a top priority, but halting the recommended screening tests will have a long-term effect in the years to come. This paper mainly focuses on the impact of cancer screening in B.P. Koirala Memorial Cancer Hospital (BPKMCH).

Cancer screening is of utmost importance in cancer prevention and control, which helps to detect cancer early even before the symptoms develop. Detecting cancer in an early stage makes cancer easier to treat as well as the outcome is very successful. BPKMCH has been conducting cancer screening programs as a separate wing. There are hospital-based cancer screening services as well as community-based cancer screening and training services. ${ }^{3}$ This pandemic has largely affected the screening services in BPKMCH especially the community-based screening. BPKMCH has been able to run the diagnostic and treatment facilities as well as screening services in the hospital taking proper precautions.

- Patients pre-screened for COVID-related symptoms before the appointment

- Measurement of body temperature before entry to the hospital

- Hand sanitizer and/or handwashing stations made available within the hospital premises

- Only one visitor pass for each patient

- Mandatory use of face mask and face shield by the patients and visitors.

- Maintaining physical distancing in the hospital as needed

- Large spacing between the patient beds

- Education to the patients and caregivers in the hospital

- Treatment including surgeries of the patient only after the COVID-19 test results

- Separate isolation for COVID-19 patients

- Virtual consultation of patients with their respective health care providers regarding follow-up and home care management

In the previous year 2075/76, BPKMCH screened 4697, 1721, and 792 clients in the community for cervical, breast, and oral cancer respectively. ${ }^{3}$ This year, the number was expected to rise as there was an increase in

Correspondence

Dr. Gambhir Shrestha, Department of Community Medicine, Maharajgunj Medical Campus, Institute of Medicine, Tribhuvan University, Kathmandu, Nepal, email:gamvir.stha@gmail.com 
community participation, but the pandemic halted the community screening services since March 2020. As the majority of cancer screening programs are carried out at the community level, there has been a major effect in the on-going screening activities of the hospital. The screening camps are mostly conducted in mass and are likely to be a hotspot for transmission of COVID-19 in the absence of an effective preventive strategy for COVID-19. There are many hurdles to be taken care of, during the camps such as overcrowding, Personal Protective Equipment (PPE) for the health professionals, and the volunteers. Meanwhile, the screening services in the community have been paused, but it needs to be started soon as the virus will be staying with us for long. We need to think of strategies to overcome these problems. The question also arises whether to conduct such kind of screening in the community or not in the future amidst the virus will remain for long until we have an effective vaccine against it. We should be assured that the community screening should not be the hot spot of transmission of the virus. The following measures should be taken care of during this pandemic to run community cancer screening services:

- Screening centers in the community should have separate entry and exit.

- A list of clients for screening should be made prior, to estimate the number to be screened in the community.

- Appointments should be scheduled at different times, which allows physical distancing between clients and avoids crowding in waiting rooms and patient care areas.

- Clients should be pre-screened for COVID-related symptoms and checked for temperature before screening procedures.

- Visitors should be limited at the entrance to avoid the crowd.

- Everyone, including clients and staff, should wear a face mask. Full PPEs should be provided to the staff, where appropriate. There should be the availability of handwashing stations and frequent handwashing and use of hand sanitizer by staff, patients, and visitors.
Cancer screening, being an elective and non-urgent service, has been unfocused in the current situation. Also, in the context of Nepal, Visual inspection with acetic acid (VIA) for cervical cancer screening and clinical breast examination is widely done in the community setting by organizing various camps at the community level, people screen for cancer due to the accessibility of services at their doorstep. However, postponing cancer screening may have a very vast impact in the years to come. Studies reported a significant increase in the number of avoidable cancer deaths to be expected because of diagnostic delays due to the COVID-19 pandemic. ${ }^{4}$ Screening services that have made a significant effort in the past few years to prevent and control cancer in Nepal may go in vain. The government should also focus on ongoing preventive and control activities. It is also equally important to continue to offer and encourage routine screening and follow up in the hospital. During this pandemic, it is not possible to offer the usual cancer screening services in the community. The government should make a guideline and implement ongoing preventive measures such as awareness campaigns and community programs including cancer screening.

\section{References}

1. World Health Organization. Coronavirus (COVID-19) events as they happen. WHO 2020. Available at: https://www.who.int/emergencies/ diseases/novel-coronavirus-2019/events-as-theyhappen

2. Thakur RK, Singh R, Shrestha G, Gautam S, Singh SK. The COVID-19 paradox and exit strategy for Nepal. Europasian J Med Sci.2020;2(Covid-19 Special Issue):1-11.

3. B.P. Koirala Memorial Cancer Hospital. Annual report 2018. BPKMCH 2019. Available at: www. bpkmch.org.np

4. Maringe C, Spicer J, Morris M, Purushotham A, Nolte E, Sullivan R, Rachet B, Aggarwal A. The impact of the COVID-19 pandemic on cancer deaths due to delays in diagnosis in England, UK: a national, population-based, modelling study. The Lancet Oncology. 2020 Aug 1;21(8):1023-34. 